\title{
AN INTEGRAL FORMULA FOR COMPACT HYPERSURFACES IN SPACE FORMS AND ITS APPLICATIONS
}

\author{
LUIS J. ALÍAS
}

(Received 5 December 2000; revised 18 March 2002)

\author{
Communicated by $\mathrm{K}$. Wysocki
}

\begin{abstract}
In this paper we establish an integral formula for compact hypersurfaces in non-flat space forms, and apply it to derive some interesting applications. In particular, we obtain a characterization of geodesic spheres in terms of a relationship between the scalar curvature of the hypersurface and the size of its Gauss map image. We also derive an inequality involving the average scalar curvature of the hypersurface and the radius of a geodesic ball in the ambient space containing the hypersurface, characterizing the geodesic spheres as those for which equality holds.
\end{abstract}

2000 Mathematics subject classification: primary $53 \mathrm{C} 40,53 \mathrm{C} 42$.

Keywords and phrases: Ricci curvature, scalar curvature, average scalar curvature, Gauss map image, geodesic sphere.

\section{Introduction}

In a recent paper, Deshmukh [1] obtained an integral formula for compact hypersurfaces in Euclidean space $\mathbb{E}^{n+1}$, and applied it to derive some interesting consequences on such hypersurfaces, including a characterization of round spherès as well as an inequality involving the average scalar curvature and the extrinsic radius of the hypersurface (we also refer the reader to $[2,3,4]$ for some other applications of this integral formula obtained by the same author). More recently, Vlachos [5] obtained an extension of this integral formula to the case of compact hypersurfaces in the non-flat space forms, namely the sphere $\mathbb{S}^{n+1}$ and the hyperbolic space $\mathbb{H}^{n+1}$, and applied it to characterize geodesic spheres in space forms.

The author was partially supported by Dirección General de Investigación (MCYT), Grant No. BFM2001-2871 and Consejería de Educación y Universidades (CARM) Programa Séneca, Grant No. PI-3/00854/FS/01.

(C) 2003 Australian Mathematical Society $1446-7887 / 03 \$ A 2.00+0.00$ 
In this paper we obtain another integral formula for compact hypersurfaces in the non-flat space forms (see Proposition 2.1), which is similar to the previous ones and allows us to derive new applications. In particular, we characterize the geodesic spheres in non-flat space forms as follows (see Corollary 3.3 and Corollary 3.4):

- Let $M^{n}$ be a compact, oriented hypersurface immersed in $\mathbb{S}^{n+1}$ such that its Ricci curvature satisfies Ric $>(n+2)(n-1)$. Let us assume that its Gauss map image $\mathbf{N}(M) \subset \mathbb{S}^{n+1}$ is contained in a tubular neighbourhood of radius $0<\eta<\pi / 2$ around an equator of $\mathbb{S}^{n+1}$. If its scalar curvature $S$ satisfies $S \leq n(n-1) / \sin ^{2}(\eta)$, then $M$ is a geodesic sphere of $\$^{n+1}$.

- Let $M^{n}$ be a compact, oriented hypersurface immersed in $\mathbb{H}^{n+1}$ such that its Ricci curvature satisfies Ric $>-(n+2)(n-1)$. Let us assume that the Gauss map image $\mathbf{N}(M) \subset \mathbb{S}_{1}^{n+1}$ is contained in a tubular neighbourhood of (timelike) radius $\eta>0$ around an equator of de Sitter space $\mathbb{S}_{1}^{n+1}$. If its scalar curvature $S$ satisfies $S \leq n(n-1) / \sinh ^{2}(\eta)$, then $M$ is a geodesic sphere of $\mathbb{H}^{n+1}$.

As another application of our integral formula, we also extend Deshmukh's inequality for the average scalar curvature to the case of compact hypersurfaces in non-flat space forms, characterizing the geodesic spheres as those for which equality holds (see Theorem 3.5 and Theorem 3.6):

- Let $M^{n}$ be a compact, oriented hypersurface immersed in $\mathbb{S}^{n+1}$ such that Ric $\geq(n+2)(n-1)$. If $M^{n}$ is contained in a geodesic ball in $\mathbb{S}^{n+1}$ of radius $0<\varrho<\pi / 2$, then

$$
\operatorname{av}(S) \geq \frac{n(n-1)}{\sin (\varrho)} .
$$

Moreover, the equality holds if and only if $M^{n}$ is a geodesic sphere of radius $\varrho$.

- Let $M^{n}$ be a compact, oriented hypersurface immersed in $\mathbb{H}^{n+1}$ such that Ric $\geq-(n+2)(n-1)$ and the scalar curvature $S$ is positive on $M$. If $M^{n}$ is contained in a geodesic ball in $\mathbb{H}^{n+1}$ of radius $Q>0$, then

$$
\operatorname{av}(S) \geq \frac{n(n-1)}{\sinh (\varrho)}
$$

Moreover, the equality holds if and only if $M^{n}$ is a geodesic sphere of radius $\varrho$.

\section{The integral formula}

Throughout this paper we will denote by $\bar{M}^{n+1}(c)$ the standard model for an $(n+1)$-dimensional, complete and simply connected space with constant sectional curvature $c, c=1,-1$. Let $\mathbb{E}_{s}^{n+2}, s=0,1$, denote the $(n+2)$-dimensional real vector 
space $\mathbb{R}^{n+2}$ endowed with the metric tensor given by

$$
\langle v, w\rangle=\sum_{i=1}^{n+1} v_{i} w_{i}+(-1)^{s} v_{n+2} w_{n+2}
$$

Then $\bar{M}^{n+1}(1)=\mathbb{S}^{n+1} \subset \mathbb{E}^{n+2}$ is the $(n+1)$-dimensional Euclidean sphere,

$$
\mathbb{S}^{n+1}=\left\{x \in \mathbb{E}^{n+2}:\langle x, x\rangle=1\right\},
$$

and $\bar{M}^{n+1}(-1)=\mathbb{H}^{n+1} \subset \mathbb{E}_{1}^{n+2}$ is the $(n+1)$-dimensional hyperbolic space,

$$
\mathbb{H}^{n+1}=\left\{x \in \mathbb{E}_{1}^{n+2}:\langle x, x\rangle=-1, x_{n+2} \geq 1\right\} \text {. }
$$

Let $\psi: M^{n} \rightarrow \bar{M}^{n+1}(c) \subset \mathbb{E}_{s}^{n+2}$ be an immersed, oriented, connected hypersurface in $\bar{M}^{n+1}(c)$, and let $\mathbf{N}$ be its globally defined unit normal field. We will denote by $\nabla^{\circ}$, $\bar{\nabla}$ and $\nabla$ the Levi-Civita connections of $\mathbb{E}_{s}^{n+2}, \bar{M}^{n+1}(c)$ and $M^{n}$, respectively. Then the Gauss and Weingarten formulas for $M^{n}$ in $\bar{M}^{n+1}(c) \subset \mathbb{E}_{s}^{n+2}$ are given respectively by

$$
\nabla_{X}^{\circ} Y=\bar{\nabla}_{X} Y-c(X, Y\rangle \psi=\nabla_{X} Y+\langle A X, Y\rangle \mathbf{N}-c\langle X, Y\rangle \psi
$$

and

$$
A(X)=-\nabla_{X}^{o} \mathbf{N}=-\bar{\nabla}_{X} \mathbf{N}
$$

for all tangent vector fields $X, Y \in \mathfrak{X}(M)$, where $A$ stands for the shape operator of $M^{n}$ in $\bar{M}^{n+1}(c)$ associated to $\mathbf{N}$.

For a fixed arbitrary vector $\mathbf{a} \in \mathbb{E}_{s}^{n+2}$, let us consider the height function $\langle\mathbf{a}, \psi\rangle$ and the function $(\mathbf{a}, \mathbf{N}\rangle$, which are defined on $M^{n}$. From (1) and (2) we know that their gradients are given by $\nabla\langle\mathbf{a}, \psi\rangle=\mathbf{a}^{\top}$, and

$$
\nabla\langle\mathbf{a}, \mathbf{N}\rangle=-A\left(\mathbf{a}^{\top}\right),
$$

where

$$
\mathbf{a}^{\top}=\mathbf{a}-\langle\mathbf{a}, \mathbf{N}\rangle \mathbf{N}-c(\mathbf{a}, \psi\rangle \psi \in \mathfrak{X}(M)
$$

By taking covariant derivative in (4) and using (1) and (2), we obtain from $\nabla^{\circ} \mathbf{a}=0$ that

$$
\nabla_{X} \mathbf{a}^{\top}=\langle\mathbf{a}, \mathbf{N}\rangle A(X)-c\langle\mathbf{a}, \psi\rangle X
$$


for $X \in \mathfrak{X}(M)$. Therefore, the Laplacian of $\langle\mathbf{a}, \psi\rangle$ is given by

$$
\Delta\langle\mathbf{a}, \psi\rangle=\sum_{i=1}^{n}\left\langle\nabla_{E_{i}} \mathbf{a}^{\top}, E_{i}\right\rangle=n H\langle\mathbf{a}, \mathbf{N}\rangle-n c\langle\mathbf{a}, \psi\rangle
$$

where $\left\{E_{1}, \ldots, E_{n}\right\}$ is a local orthonormal frame on $M^{n}$ and $H=\operatorname{tr}(A) / n$ is the mean curvature function of $M^{n}$, and

$$
\begin{aligned}
\frac{1}{2} \Delta\langle\mathbf{a}, \psi\rangle^{2} & =\langle\mathbf{a}, \psi\rangle \Delta\langle\mathbf{a}, \psi\rangle+|\nabla\langle\mathbf{a}, \psi\rangle|^{2} \\
& =n H\langle\mathbf{a}, \psi\rangle\langle\mathbf{a}, \mathbf{N}\rangle-n c\langle\mathbf{a}, \psi\rangle^{2}+\left|\mathbf{a}^{\top}\right|^{2} .
\end{aligned}
$$

On the other hand, from (3) the Laplacian of $\langle\mathbf{a}, \mathbf{N}\rangle$ is given by

$$
\Delta\langle\mathbf{a}, \mathbf{N}\rangle=-\sum_{i=1}^{n}\left\langle\nabla_{E_{i}}\left(A \mathbf{a}^{\top}\right), E_{i}\right\rangle=-\sum_{i=1}^{n}\left\langle\left(\nabla_{E_{i}} A\right) \mathbf{a}^{\top}, E_{i}\right\rangle-\sum_{i=1}^{n}\left\langle A\left(\nabla_{E_{i}} \mathbf{a}^{\top}\right), E_{i}\right\rangle .
$$

Using now the Codazzi equation, $\left(\nabla_{X} A\right)\left(\mathbf{a}^{\top}\right)=\left(\nabla_{\mathbf{a}^{\top}} A\right)(X)$, jointly with (5) we conclude that

$$
\begin{aligned}
\Delta\langle\mathbf{a}, \mathbf{N}\rangle & =-\operatorname{tr}\left(\nabla_{\mathbf{a}} \cdot A\right)-\langle\mathbf{a}, \mathbf{N}\rangle|A|^{2}+n c H\langle\mathbf{a}, \psi\rangle \\
& =-n\langle\nabla H, \mathbf{a}\rangle-\langle\mathbf{a}, \mathbf{N}\rangle|A|^{2}+n c H\langle\mathbf{a}, \psi\rangle
\end{aligned}
$$

where $|A|^{2}=\operatorname{tr}\left(A^{2}\right)$, and

(8) $\frac{1}{2} \Delta\langle\mathbf{a}, \mathbf{N}\rangle^{2}=-n\langle\mathbf{a}, \mathbf{N}\rangle\langle\nabla H, \mathbf{a}\rangle-\langle\mathbf{a}, \mathbf{N}\rangle^{2}|A|^{2}+n c H\langle\mathbf{a}, \psi\rangle\langle\mathbf{a}, \mathbf{N}\rangle+\left|A\left(\mathbf{a}^{\top}\right)\right|^{2}$.

Now, let us recall that the Ricci curvature of $M^{n}$ is given by

$$
\operatorname{Ric}(X, Y)=(n-1) c\langle X, Y\rangle+n H\langle A X, Y\rangle-\langle A X, A Y\rangle
$$

for $X, Y \in \mathfrak{X}(M)$. This allows us to write

$$
\left|A\left(\mathbf{a}^{\top}\right)\right|^{2}=(n-1) c\left|\mathbf{a}^{\top}\right|^{2}-n H\langle\nabla(\mathbf{a}, \mathbf{N}), \mathbf{a}\rangle-\operatorname{Ric}\left(\mathbf{a}^{\top}, \mathbf{a}^{\top}\right),
$$

so that (8) becomes

$$
\begin{aligned}
\frac{1}{2} \Delta\langle\mathbf{a}, \mathbf{N}\rangle^{2}= & -n\langle\nabla(H\langle\mathbf{a}, \mathbf{N}\rangle), \mathbf{a}\rangle-\langle\mathbf{a}, \mathbf{N}\rangle^{2}|A|^{2}+n c H\langle\mathbf{a}, \psi\rangle\langle\mathbf{a}, \mathbf{N}\rangle \\
& -\operatorname{Ric}\left(\mathbf{a}^{\top}, \mathbf{a}^{\top}\right)+(n-1) c\left|\mathbf{a}^{\top}\right|^{2} .
\end{aligned}
$$

Since

$$
\begin{aligned}
\langle\nabla(H\langle\mathbf{a}, \mathbf{N}\rangle), \mathbf{a}\rangle & =\langle\nabla(H\langle\mathbf{a}, \mathbf{N}\rangle), \nabla\langle\mathbf{a}, \psi\rangle\rangle \\
& =\operatorname{div}(H\langle\mathbf{a}, \mathbf{N}\rangle \nabla\langle\mathbf{a}, \psi\rangle)-H\langle\mathbf{a}, \mathbf{N}\rangle \Delta\langle\mathbf{a}, \psi\rangle,
\end{aligned}
$$


we obtain from (9), using (6), that

$$
\begin{aligned}
\frac{1}{2} \Delta\langle\mathbf{a}, \mathbf{N}\rangle^{2}= & -n \operatorname{div}(H\langle\mathbf{a}, \mathbf{N}\rangle \nabla\langle\mathbf{a}, \psi\rangle)-\operatorname{Ric}\left(\mathbf{a}^{\top}, \mathbf{a}^{\top}\right)+(n-1) c\left|\mathbf{a}^{\top}\right|^{2} \\
& +\left(n^{2} H^{2}-|A|^{2}\right)\langle\mathbf{a}, \mathbf{N}\rangle^{2}-n(n-1) c H\langle\mathbf{a}, \psi\rangle\langle\mathbf{a}, \mathbf{N}\rangle .
\end{aligned}
$$

Finally, using (7) and the fact that $\langle\mathbf{a}, \mathbf{a}\rangle=\left|\mathbf{a}^{\top}\right|^{2}+\langle\mathbf{a}, \mathbf{N}\rangle^{2}+c\langle\mathbf{a}, \psi\rangle^{2}$ we conclude that

$$
\begin{aligned}
\frac{1}{2} & \Delta\langle\mathbf{a}, \mathbf{N}\rangle^{2}+n \operatorname{div}(H\langle\mathbf{a}, \mathbf{N}\rangle \nabla\langle\mathbf{a}, \psi\rangle)+\frac{c(n-1)}{2} \Delta\langle\mathbf{a}, \psi\rangle^{2} \\
& =-\operatorname{Ric}\left(\mathbf{a}^{\top}, \mathbf{a}^{\top}\right)+(n+2)(n-1) c\left|\mathbf{a}^{\top}\right|^{2}+S\langle\mathbf{a}, \mathbf{N}\rangle^{2}-n(n-1) c\langle\mathbf{a}, \mathbf{a}\rangle,
\end{aligned}
$$

where $S$ stands for the scalar curvature of $M^{n}$,

$$
S=\operatorname{tr}(\text { Ric })=n(n-1) c+n^{2} H^{2}-|A|^{2} .
$$

Integrating now (10) on $M^{n}$, the divergence theorem implies our integral formula.

PROPOSITION 2.1. Let $\psi: M^{n} \rightarrow \bar{M}^{n+1}(c) \subset \mathbb{E}_{s}^{n+2}$ be a compact, oriented hypersurface immersed in $\bar{M}^{n+1}(c), c=1,-1$, and let $\mathbf{a} \in \mathbb{E}_{s}^{n+2}$ be a fixed arbitrary vector. Then, if Ric and $S$ denote respectively the Ricci curvature and the scalar curvature of $M^{n}$, it holds that

$$
\begin{gathered}
\int_{M}\left(\operatorname{Ric}\left(\mathbf{a}^{\top}, \mathbf{a}^{\top}\right)-(n+2)(n-1) c\left|\mathbf{a}^{\top}\right|^{2}\right) \mathrm{d} V \\
=\int_{M}\left(S\langle\mathbf{a}, \mathbf{N}\rangle^{2}-n(n-1) c(\mathbf{a}, \mathbf{a}\rangle\right) \mathrm{d} V,
\end{gathered}
$$

where $\mathrm{d} V$ is the $n$-dimensional volume element of $M^{n}$ with respect to the induced metric and the chosen orientation.

\section{Applications}

In this section we will derive some consequences and applications of our integral formula (11). As a first application, we obtain the following characterization of geodesic spheres in $\bar{M}^{n+1}(c)$, which is inspired in the characterization of geodesic spheres given by Deshmukh [1, Theorem 3] in the Euclidean case and by Vlachos [5, Theorem 2] in the spherical and hyperbolic cases.

THEOREM 3.1. Let $\psi: M^{n} \rightarrow \bar{M}^{n+1}(c) \subset \mathbb{E}_{s}^{n+2}$ be a compact, oriented hypersurface immersed in $\bar{M}^{n+1}(c)$ such that its Ricci curvature satisfies Ric $>c(n+2)(n-1)$. If there exists a point a $\in \bar{M}^{n+1}(c)$ for which the scalar curvature $S$ satisfies $S\langle\mathbf{a}, \mathbf{N}\rangle^{2} \leq n(n-1)$, then $\psi(M)$ is a geodesic sphere of $\bar{M}^{n+1}(c)$ centered at the point $\mathbf{a}$. 
PROOF. It follows from the assumption on the Ricci curvature that

$$
\operatorname{Ric}\left(\mathbf{a}^{\top}, \mathbf{a}^{\top}\right)-(n+2)(n-1) c\left|\mathbf{a}^{\top}\right|^{2} \geq 0
$$

with equality if and only if $\mathbf{a}^{\top}=\nabla\langle\mathbf{a}, \psi\rangle$ vanishes everywhere. Therefore, using our integral formula (11) we obtain that

$$
\int_{M}\left(S\langle\mathbf{a}, \mathbf{N})^{2}-n(n-1)\right) \mathrm{d} V \geq 0,
$$

with equality if and only if $\langle\mathbf{a}, \psi\rangle$ is constant on $M^{n}$. On the other hand, from the assumption on the scalar curvature we also have

$$
\int_{M}\left(S\langle\mathbf{a}, \mathbf{N}\rangle^{2}-n(n-1)\right) \mathrm{d} V \leq 0,
$$

which gives the equality in (12) and implies that

$$
\psi(M)=\left\{x \in \bar{M}^{n+1}(c):\langle\mathbf{a}, x\rangle=b\right\}
$$

for a real constant $b$. If $c=1$, then $b=\cos (\varrho)$ with $0<\varrho<\pi$ and $\psi(M)$ is a geodesic sphere in $\$^{n+1}$ centered at a with radius $\varrho$. If $c=-1$, then $b=\cosh (\varrho)$ with $\varrho>0$ and $\psi(M)$ is a geodesic sphere in $\mathbb{H}^{n+1}$ centered at a with radius $\varrho$.

In the case where $c=1$ (spherical hypersurfaces) the result above can be paraphrased in a more geometric statement as follows. Since $c=1$, the Gauss map $\mathbf{N}$ can be regarded as a map $\mathbf{N}: M^{n} \rightarrow \mathbb{S}^{n+1}$, and for every $\mathbf{a} \in \mathbb{S}^{n+1}$ the function $\langle\mathbf{a}, \mathbf{N}\rangle$ gives a measure of the spherical distance between a and $\mathbf{N}$. Actually, for every $p \in M$ we know that the spherical distance between a and $\mathbf{N}(p)$ is given by

$$
\varrho_{\mathbf{a}}(p)=\operatorname{arcos}(\langle\mathbf{a}, \mathbf{N}(p)\rangle) .
$$

For instance, when $M=\mathbb{S}^{n}(\mathbf{a}, \eta) \subset \mathbb{S}^{n+1}$ is a geodesic sphere in $\mathbb{S}^{n+1}$ centered at a with radius $\eta, 0<\eta<\pi$, then it is easy to see that $\varrho_{\mathbf{a}}=|\pi / 2-\eta|$ is constant on $M$. Moreover, $M$ is a round $n$-sphere of radius $\sin (\eta)$ and its scalar curvature is given by $S=n(n-1) / \sin ^{2}(\eta)=n(n-1) / \cos ^{2}\left(\varrho_{\mathrm{a}}\right)$. Now, Theorem 3.1 can be rewritten in terms of the spherical distance as follows.

COROLLARY 3.2. Let $\psi: M^{n} \rightarrow \mathbb{S}^{n+1} \subset \mathbb{E}^{n+2}$ be a compact, oriented hypersurface immersed in $\mathbb{S}^{n+1}$ such that Ric $>(n+2)(n-1)$. For a given a $\in \mathbb{S}^{n+1}$ and $p \in M$, let us denote by $Q_{\mathbf{a}}(p)$ the spherical distance between $\mathbf{a}$ and the image of $p$ under the Gauss map $\mathbf{N}$. If there exists a point $\mathbf{a} \in \mathbb{S}^{n+1}$ for which

$$
\cos ^{2}\left(\varrho_{\mathrm{a}}\right) \leq \frac{n(n-1)}{S}
$$

then $\psi(M)$ is a geodesic sphere of $\S^{n+1}$ centered at the point $\mathbf{a}$. 
As another consequence of this, we have the following.

COROLLARY 3.3. Let $\psi: M^{n} \rightarrow \mathbb{S}^{n+1} \subset \mathbb{E}^{n+2}$ be a compact, oriented hypersurface immersed in $\mathbb{S}^{n+1}$ such that $\mathrm{Ric}>(n+2)(n-1)$. Let us assume that its Gauss map image $\mathbf{N}(M) \subset \mathbb{S}^{n+1}$ is contained in a tubular neighbourhood of radius $0<\eta<\pi / 2$ around an equator of $\mathbb{S}^{n+1}$. If its scalar curvature $S$ satisfies

$$
S \leq \frac{n(n-1)}{\sin ^{2}(\eta)},
$$

then $\psi(M)$ is a geodesic sphere of $\mathbb{\$}^{n+1}$ parallel to that equator.

PROOF. Let us assume that the spherical image of $M^{n}$ is contained in a tubular neighbourhood $\mathscr{U}(\mathbf{a}, \eta)$ in $\mathbb{S}^{n+1}$ of radius $0<\eta<\pi / 2$ around the equator given by $\mathbb{S}^{n+1} \cap \mathbf{a}^{\perp}$. Observe that

$$
\mathscr{U}(\mathbf{a}, \eta)=\left\{x \in \mathbb{S}^{n+1}:-\sin (\eta) \leq\langle\mathbf{a}, x\rangle \leq \sin (\eta)\right\},
$$

so that $\langle\mathbf{a}, \mathbf{N}\rangle^{2} \leq \sin ^{2}(\eta)$ on $M^{n}$. At each point $p \in M^{n}$ we have that $0<S(p) \leq$ $n(n-1) / \sin ^{2}(\eta)$ and, by the assumption on $S$,

$$
S(p)\langle\mathbf{a}, \mathbf{N}(p)\rangle^{2} \leq S(p) \sin ^{2}(\eta) \leq n(n-1),
$$

so that we may apply Theorem 3.1.

On the other hand, when $c=-1$ (hyperbolic hypersurfaces) the Gauss map of $M^{n}$ can be regarded as a map $\mathbf{N}: M^{n} \rightarrow \mathbb{S}_{1}^{n+1}$, where $\mathbb{S}_{1}^{n+1}$ denotes the $(n+1)$-dimensional unitary de Sitter space, that is,

$$
\mathbb{S}_{1}^{n+1}=\left\{x \in \mathbb{E}_{1}^{n+2}:\langle x, x\rangle=1\right\} .
$$

As is well known, for $n \geq 2$ de Sitter space is the standard simply connected Lorentzian space form with constant sectional curvature 1 . In this case, if $\mathbf{a} \in \mathbb{H}^{n+1}$, the intersection $\mathbb{S}_{1}^{n+1} \cap \mathbf{a}^{\perp}$ defines a round $n$-sphere of radius 1 which is a totally geodesic spacelike hypersurface in $\$_{1}^{n+1}$. We will refer to that sphere as the equator of de Sitter space determined by a. In this terms, when $c=-1$ Theorem 3.1 can be rewritten as follows.

COROLLARY 3.4. Let $\psi: M^{n} \rightarrow \mathbb{H}^{n+1} \subset \mathbb{E}_{1}^{n+2}$ be a compact, oriented hypersurface immersed in $\mathbb{H}^{n+1}$ such that Ric $>-(n+2)(n-1)$. Let us assume that the Gauss map image $\mathbf{N}(M) \subset \mathbb{S}_{1}^{n+1}$ is contained in a tubular neighbourhood of (timelike) radius $\eta>0$ around an equator of $\mathbb{S}_{1}^{n+1}$ determined by $\mathbf{a} \in \mathbb{H}^{n+1}$. If its scalar curvature $S$ satisfies

$$
S \leq \frac{n(n-1)}{\sinh ^{2}(\eta)}
$$

then $\psi(M)$ is a geodesic sphere of $\mathbb{H}^{n+1}$ centered at a. 
The proof is similar to that of Corollary 3.3 by observing that the tubular neighbourhood $\mathscr{U}(\mathbf{a}, \eta)$ in $\mathbb{S}_{1}^{n+1}$ of (timelike) radius $\eta$ around the equator $\mathbb{S}_{1}^{n+1} \cap \mathbf{a}^{\perp}$ is given by

$$
\mathscr{U}(\mathbf{a}, \eta)=\left\{x \in \mathbb{S}_{1}^{n+1}:-\sinh (\eta) \leq\langle\mathbf{a}, x\rangle \leq \sinh (\eta)\right\} .
$$

On the other hand, in [1, Theorem 1, Theorem 2], Deshmukh gave an interesting inequality involving the extrinsic radius of a non-negatively Ricci curved compact hypersurface in Euclidean space $M^{n}$ and its average scalar curvature av $(S)$, which is defined by the Einstein functional

$$
\operatorname{av}(S)=\frac{1}{\operatorname{vol}(M)} \int_{M} S \mathrm{~d} V .
$$

In this paper, and as another application of our integral formula (11), we extend Deshmukh's inequality to the case of compact hypersurfaces in the other space forms, also characterizing when the equality holds, as follows.

THEOREM 3.5. Let $\psi: M^{n} \rightarrow \mathbb{S}^{n+1} \subset \mathbb{E}^{n+2}$ be a compact, oriented hypersurface immersed in $\mathbb{S}^{n+1}$ such that Ric $\geq(n+2)(n-1)$. If the image $\psi(M) \subset \mathbb{S}^{n+1}$ is contained in a geodesic ball in $\mathbb{S}^{n+1}$ of radius $0<\varrho<\pi / 2$, then

$$
\operatorname{av}(S) \geq \frac{n(n-1)}{\sin (\varrho)} .
$$

Moreover, the equality holds if and only if $M^{n}$ is a geodesic sphere of radius $\varrho$.

THEOREM 3.6. Let $\psi: M^{n} \rightarrow \mathbb{H}^{n+1} \subset \mathbb{E}_{1}^{n+2}$ be a compact, oriented hypersurface immersed in $\mathbb{H}^{n+1}$ such that $\mathrm{Ric} \geq-(n+2)(n-1)$ and the scalar curvature $S$ is positive on $M$. If the image $\psi(M) \subset \mathbb{H}^{n+1}$ is contained in a geodesic ball in $\mathbb{H}^{n+1}$ of radius $\varrho>0$, then

$$
\operatorname{av}(S) \geq \frac{n(n-1)}{\sinh (\varrho)}
$$

Moreover, the equality holds if and only if $M^{n}$ is a geodesic sphere of radius $\varrho$.

COROLlaRY 3.7. Let $\psi: M^{n} \rightarrow \mathbb{H}^{n+1} \subset \mathbb{E}_{1}^{n+2}$ be a positively Ricci curved, compact, oriented hypersurface immersed in $\mathbb{H}^{n+1}$. If the image $\psi(M) \subset \mathbb{H}^{n+1}$ is contained in a geodesic ball in $\mathbb{H}^{n+1}$ of radius $\varrho>0$, then

$$
\operatorname{av}(S) \geq \frac{n(n-1)}{\sinh (\varrho)} .
$$

Moreover, the equality holds if and only if $M^{n}$ is a geodesic sphere of radius $\varrho$. 
ProOF OF THEOREM 3.5. Let us assume that $\psi(M)$ is contained in a geodesic ball $\mathscr{B}(\mathbf{a}, \varrho)$ in $\mathbb{S}^{n+1}$ of radius $0<\varrho<\pi / 2$ centered at a point $\mathbf{a} \in \mathbb{S}^{n+1}$. Recall that

$$
\mathscr{B}(\mathbf{a}, \varrho)=\left\{x \in \mathbb{S}^{n+1}: 0<\cos (\varrho) \leq\langle\mathbf{a}, x\rangle \leq 1\right\},
$$

so that $\langle\mathbf{a}, \psi\rangle^{2} \geq \cos ^{2}(\varrho)$, and

$$
\langle\mathbf{a}, \mathbf{N}\rangle^{2}=1-\langle\mathbf{a}, \psi\rangle^{2}-|\mathbf{a}|^{2} \leq 1-\cos ^{2}(\varrho)=\sin ^{2}(\varrho)
$$

on $M$, with equality if and only if $\mathbf{a}^{\top}=0$ and $\langle\mathbf{a}, \psi\rangle=\cos (\varrho)$ is constant on $M$. Since $S>0$ on $M$, then

$$
S \sin ^{2}(\varrho) \geq S(\mathbf{a}, \mathbf{N})^{2},
$$

so that from (11) we obtain

$$
\begin{aligned}
\sin ^{2}(\varrho) & \int_{M} S \mathrm{~d} V-n(n-1) \operatorname{vol}(M) \\
= & \int_{M}\left(S \sin ^{2}(\varrho)-n(n-1)\right) \mathrm{d} V \geq \int_{M}\left(S\langle\mathbf{a}, \mathbf{N}\rangle^{2}-n(n-1)\right) \mathrm{d} V \\
= & \int_{M}\left(\operatorname{Ric}\left(\mathbf{a}^{\top}, \mathbf{a}^{\top}\right)-(n+2)(n-1)\left|\mathbf{a}^{\top}\right|^{2}\right) \mathrm{d} V \geq 0 .
\end{aligned}
$$

That is,

$$
\operatorname{av}(S)=\frac{1}{\operatorname{vol}(M)} \int_{M} S \mathrm{~d} V \geq \frac{n(n-1)}{\sin ^{2}(\varrho)} .
$$

Moreover, equality holds if and only if $\langle\mathbf{a}, \psi\rangle=\cos (\varrho)$ is constant on $M$, which means that $M$ is a geodesic sphere of radius $\varrho$ centered at $\mathbf{a}$.

The proof of Theorem 3.6 is similar to that of Theorem 3.5 by observing that the geodesic ball $\mathscr{B}(\mathbf{a}, \varrho)$ in $\mathbb{H}^{n+1}$ of radius $\varrho$ centered at a point $\mathbf{a}$ is given by

$$
\mathscr{B}(\mathbf{a}, \varrho)=\left\{x \in \mathbb{H}^{n+1}: 1 \leq\langle\mathbf{a}, x\rangle \leq \cosh (\varrho)\right\},
$$

and using now that $\langle\mathbf{a}, \mathbf{N}\rangle^{2}=\langle\mathbf{a}, \psi\rangle^{2}-1-|\mathbf{a}|^{2}$.

\section{References}

[1] S. Deshmukh, 'An integral formula for compact hypersurfaces in a Euclidean space and its applications', Glasgow Math. J. 34 (1992), 309-311.

[2] — , 'Hypersurfaces of non-negative Ricci curvature in a Euclidean space', J. Geom. 45 (1992), 48-50. 
[3] — - 'Isometric immersion of a compact Riemannian manifold into a Euclidean space', Bull. Austral. Math. Soc. 46 (1992), 177-178.

[4] —_, 'Compact hypersurfaces in a Euclidean space', Quart. J. Math. Oxford Ser. (2) 49 (1998), 35-41.

[5] T. Vlachos, 'An integral formula for hypersurfaces in space forms', Glasgow Math. J. 37 (1995), 337-341.

Departamento de Matemáticas

Universidad de Murcia

E-30100 Espinardo, Murcia

Spain

e-mail: ljalias@um.es 